\title{
Fisiologia do estresse calórico e a utilização de eletrólitos em frangos de corte
}

\author{
Heat stress physiology and eletrolites for broilers
}

Sebastião Aparecido Borges ${ }^{1}$ Alex Maiorka ${ }^{2}$ Ana Vitória Fischer da Silva ${ }^{3}$

\section{- REVISÃO BIBLIOGRÁFICA -}

RESUMO

Várias alterações metabólicas e fisiológicas são desencadeadas em frangos de corte submetidos a altas temperaturas ambientais, o que pode acarretar em grandes perdas no desempenho e na imunocompetência destas aves. Além das técnicas de controle ambiental estarem sendo freqüentemente empregadas para a redução do impacto negativo do estresse calórico sobre o desempenho das aves, outras medidas estão constantemente sendo estudadas. Nos últimos anos, o manejo nutricional adequado também tem demonstrado ser efetivo como medida preventiva para o estresse calórico, pois o funcionamento do sistema termorregulador do frango (produção de calor, rotas evaporativas e não evaporativas de dissipação de calor) pode ser influenciado pela dieta, em especial, o estabelecimento de adequados balanços eletrolíticos, devido a sua importância fisiológica no mecanismo do estresse calórico. Assim, os mecanismos nutricionais devem ser reavaliados como uma ferramenta no controle desta disfunção metabólica das aves.

Palavras-chave: aves, balanço eletrolitico, estresse calórico, parâmetros sangüineos.

\section{ABSTRACT}

High ambient temperature could result in numerous physiological and metabolic perturbations on broilers chicken with consequently adversely impact in broilers performance and immune response. Though the environmental control technique have been frequently useful for reduce the negative impact of heat stress in poultry performance, other alternative have been studied. Lately, the nutritional manipulation is also used for reducing the heat stress, once the most part of thermobalance components of broiler (heat production, evaporative and nonevaporative heat dissipation routes) could be manipulated though the diet. Specially, the eletrolitic balance have fundamental importance in physiological stress mechanism and for this way should be considered as a tool in control of this metabolic dysfunction in birds.

Key words: blood parameters, eletrolitic balance, heat stress, poultry.

\section{INTRODUÇÃO}

A evolução da avicultura resultou em um frango de corte precoce e com grande eficiência para converter diferentes alimentos em proteína animal. Apesar disso, uma série de problemas metabólicos e de manejo têm surgido, destacando-se entre eles o estresse calórico. A susceptibilidade das aves ao estresse calórico aumenta à medida que o binômio umidade relativa e temperatura ambiente ultrapassam a zona de conforto térmico, dificultando assim a dissipação de calor, incrementando conseqüentemente a temperatura corporal da ave, com efeito negativo sobre o desempenho.

Algumas medidas podem ser tomadas para minimizar as perdas decorrentes do estresse calórico, podendo-se citar, entre outras, a utilização de ventiladores e nebulizadores, manipulação da proteína e energia da dieta, aclimatação das aves, utilização de antitérmicos, ácido ascórbico, eletrólitos, manejo do arraçoamento e o manejo da água de bebida. Uma das conseqüências do estresse é a mudança no equilíbrio ácido-base com o aparecimento da alcalose respiratória. Assim, um dos métodos usados para o controle do estresse calórico é a manipulação química do equilíbrio ácido-base das aves através de compostos

\footnotetext{
${ }^{1}$ Aluno de Pós-graduação (Doutorado) em Zootecnia, Faculdade de Ciências Agrárias e Veterinárias, UNESP/Jaboticabal.

${ }^{2}$ Professor do Departamento de Zootecnia, Setor de Ciências Agrárias, Universidade Federal do Paraná (UFPr). E-mail: Amaiorka@ufpr.br.

${ }^{3}$ Professor do Departamento de Fisiologia, UFPr, Setor de Ciências Biológicas, Centro Politécnico, Jardim das Américas, Fone (0xx41) 3663144 R.136, 81531-970, Curitiba-PR. Email:avitoria@bio.ufpr.br. Autor para Correspondência.
} 
como bicarbonato de sódio $\left(\mathrm{NaHCO}_{3}\right)$, cloreto de potássio $(\mathrm{KCl})$, cloreto de cálcio $\left(\mathrm{CaCl}_{2}\right)$ e cloreto de amônia $\left(\mathrm{NH}_{4} \mathrm{Cl}\right)$ na água e/ou na ração (BORGES, 1997). O objetivo deste texto é fazer uma abordagem sobre os efeitos prejudiciais do estresse calórico nas aves e discutir algumas técnicas que os minimizem.

\section{Fisiologia do Estresse Calórico nas Aves}

As aves, sendo animais homeotermos, dispõem de um centro termorregulador, localizado no hipotálamo, capaz de controlar a temperatura corporal através de mecanismos fisiológicos e respostas comportamentais, mediante a produção e liberação de calor, determinando assim a manutenção da temperatura corporal normal (MACARI et al., 1994).

Entre as respostas fisiológicas compensatórias das aves, quando expostas ao calor, inclui-se a vasodilatação periférica, resultando em aumento na perda de calor não evaporativo. Assim, na tentativa de aumentar a dissipação do calor, a ave consegue aumentar a área superficial, mantendo as asas afastadas do corpo, eriçando as penas e intensificando a circulação periférica. A perda de calor não evaporativo pode também ocorrer com o aumento da produção de urina, se esta perda de água for compensada pelo maior consumo de água fria.

Outra resposta fisiológica é o aumento na taxa respiratória, resultando em perdas excessivas de dióxido de carbono $\left(\mathrm{CO}_{2}\right)$. Assim, a pressão parcial de $\mathrm{CO}_{2}(\mathrm{pCO} 2)$ diminui, levando à queda na concentração de ácido carbônico $\left(\mathrm{H}_{2} \mathrm{CO}_{3}\right)$ e hidrogênio $\left(\mathrm{H}^{+}\right)$. Em resposta, os rins aumentam a excreção de $\mathrm{HCO}_{3}^{-}$e reduzem a excreção de $\mathrm{H}^{+}$na tentativa de manter o equilíbrio ácido-base da ave (Figura 1). Esta alteração do equilíbrio ácido-base é denominada de alcalose respiratória.

\section{Estresse Calórico X Parâmetros Sangüíneos}

O sistema sangüíneo é particularmente sensível às mudanças de temperatura e se constitui em um importante indicador das respostas fisiológicas da ave a agentes estressores. Alterações quantitativas e morfológicas nas células sangüíneas são associadas ao estresse calórico, traduzidas por variações nos valores do hematócrito, número de leucócitos circulantes, conteúdo de eritrócitos e teor de hemoglobina no eritrócito.

No estresse calórico, ocorre aumento no hematócrito, podendo ser justificado por um acréscimo no número de hemácias. A relação heterófilo/linfócito é alterada como conseqüência do aumento de heterófilo e redução de linfócito, sendo que a relação heterófilo/linfócito tem sido proposta como um índice sensível de estresse crônico em frangos de corte. Outra resposta é o aumento da concentração de glicose em resposta direta à maior secreção de adrenalina, noradrenalina e glicocorticoides (BORGES, 1997; BORGES, 2001).

\section{Estresse Calórico X Eletrólitos}

Eletrólito pode ser definido como uma substância química, que se dissocia nos seus constituintes iônicos, tendo como função fisiológica principal a manutenção do equilíbrio ácido-base corporal. O sódio $\left(\mathrm{Na}^{+}\right)$, o potássio $\left(\mathrm{K}^{+}\right)$e o cloro $\left(\mathrm{Cl}^{-}\right.$ ) são íons fundamentais na manutenção da pressão osmótica e equilíbrio ácido-base dos líquidos corporais. Assim, os efeitos do balanço iônico da dieta no desempenho de frangos de corte podem estar relacionados com as variações no balanço ácido-base (MONGIN, 1981).

$\mathrm{O} \mathrm{K}^{+}$é o principal cátion do fluído intracelular, enquanto que o $\mathrm{Na}^{+}$e o $\mathrm{Cl}^{-}$são os principais íons do fluído extracelular. A osmorregulação é conseguida pela homeostasia destes íons intra e extra celular. Em condições ótimas, os conteúdos de água e eletrólitos são mantidos dentro de limites estreitos. Mas a perda de eletrólitos $\left(\mathrm{Na}^{+}\right.$ ou $\mathrm{K}^{+}$), sem alteração no conteúdo de água do corpo, reduz a osmolalidade destes fluídos.

$\mathrm{O} \mathrm{K}^{+}$está envolvido em muitos processos metabólicos, incluindo o antagonismo arginina-lisina, condução nervosa, excitação, contração muscular, síntese de proteínas teciduais, manutenção da homeostasia intracelular, reações enzimáticas, balanço osmótico e equilíbrio ácido-base. Conseqüentemente, mudanças na homeostase de $\mathrm{K}^{+}$podem afetar as funções celulares. Estudos com desidratação térmica, seguida de reidratação em humanos, demonstraram que o grau de déficit de água no fluído intracelular foi

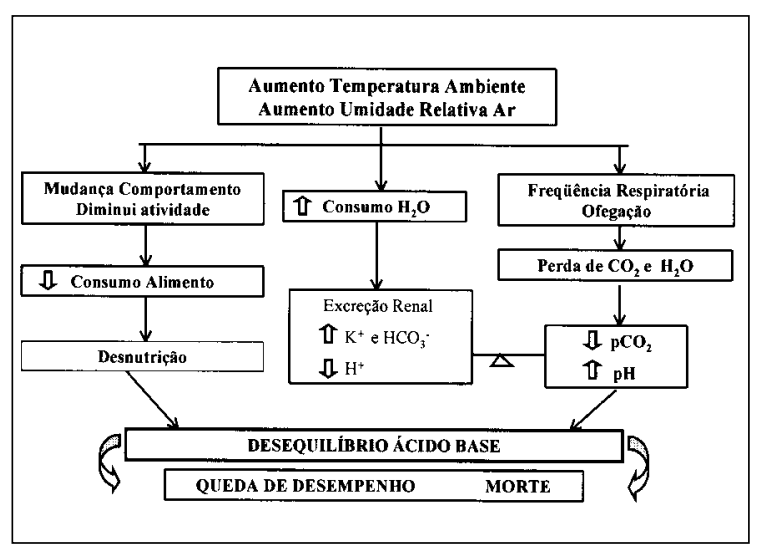

Ciência Rural, v. 33, n. 5, set-out, 2003. 
associada à perda de $\mathrm{K}^{+}$intracelular e o déficit de fluído extracelular foi ligada à perda de $\mathrm{Na}^{+}$plasmático. $\mathrm{O}$ grau de reidratação intracelular foi determinado pela restauração do $\mathrm{K}^{+}$(NOSE et al., 1988).

A alcalose respiratória, em mamíferos, provoca a redução da competição entre $\mathrm{H}^{+}$e $\mathrm{K}^{+}$para excreção urinária e, portanto, aumenta a perda de $\mathrm{K}^{+}$ na urina. $\mathrm{O}$ excesso de íons $\mathrm{K}^{+}$compete com os ânions tampões do líquido tubular renal, impedindo a remoção do $\mathrm{H}^{+}$, sendo este reabsorvido, podendo levar a uma acidose (BACILA, 1980). Tal mecanismo, quando presente em frangos, pode aumentar a necessidade de $\mathrm{K}^{+}$durante o período de estresse calórico. Existem evidências recentes de que as células intercaladas do ducto coletor, secretoras de ácidos, também secretam $\mathrm{H}^{+}$e este processo é aumentado pela hipocalemia e parece ser um importante contribuinte para acidificação renal (CUNNINGHAM, 1999).

Os níveis de $\mathrm{Na}^{+}, \mathrm{K}^{+}$e $\mathrm{Cl}^{-}$do plasma são afetados pelo estresse calórico. A concentração de $\mathrm{K}^{+}$ e $\mathrm{Na}^{+}$diminui à medida que a temperatura aumenta (BORGES, 1997), enquanto que o $\mathrm{Cl}^{-}$aumenta (BELAY \& TEETER, 1993).

$\mathrm{O}$ aumento no $\mathrm{Cl}^{-}$deprime a excreção de $\mathrm{H}^{+}$e a reabsorção de $\mathrm{HCO}_{3}$ pelos rins. Isto poderia contribuir com uma acidificação do sangue, e esta parece ser uma resposta apropriada à alcalose. Entretanto, há que se considerar o tempo de estresse já que, SALVADOR et al. (1999) observaram redução nos níveis séricos de $\mathrm{Cl}^{-}$quando submeteram frangos de corte à estresse crônico durante uma semana (42 a 49 dias de idade).

Os níveis séricos de $\mathrm{K}^{+}$também são influenciados pelo calor. A excreção de $\mathrm{K}^{+}$é influenciada por fatores hormonais (aldosterona, hormônio antidiurético - ADH e deoxicorticosterona), equilíbrio ácido-base e balanço de cátions. A taxa de excreção de $\mathrm{K}^{+}$pela urina é variável, estando ligada à concentração plasmática de $\mathrm{Na}^{+}$e ao estado de hidratação da ave, sendo que as perdas podem ser causadas por um aumento no consumo de água, já que o gradiente osmótico favorece o movimento de água do fluído intracelular para urina, podendo carrear o $\mathrm{K}^{+}$. O aumento na ingestão de $\mathrm{K}^{+}$resulta em maior perda urinária, sendo que a ave tem pouca capacidade de conservar o $\mathrm{K}^{+}$corporal. O nível sérico de $\mathrm{K}^{+}$ diminui durante o estresse (BORGES, 1997; SALVADOR et al., 1999). A diminuição dos níveis plasmáticos de $\mathrm{K}^{+}$é atribuído a um aumento na excreção deste íon durante o estresse crônico e um aumento do $\mathrm{K}^{+}$intracelular comumente encontrado durante o estresse agudo. Em humanos, a hipercalemia pode resultar em acidose metabólica, tanto pela redução da excreção de amônia como pela limitação da reabsorção de carbonato pelos rins (AITBOULAHSEN et al., 1995).

\section{Medidas Nutricionais de Controle do Estresse Calórico Utilização de sais}

A utilização de sais via água de bebida ou ração é uma alternativa freqüentemente empregada pelos produtores de frangos de corte para reduzir as perdas decorrentes do estresse calórico. Entre os principais sais utilizados destacam-se o cloreto de potássio $(\mathrm{KCl})$ e o bicarbonato de sódio $\left(\mathrm{NaHCO}_{3}\right)$.

$\mathrm{O} \mathrm{K}^{+}$está presente em abundância na maioria dos ingredientes das dietas dos animais, ao contrário do $\mathrm{Na}^{+}$, que está presente em quantidades nutricionalmente inadequadas nos alimentos naturais destinados à nutrição animal.

A suplementação de $\mathrm{KCl}$ na ração e/ou na água de bebida das aves tem sido proposta como uma forma de minimizar as conseqüências das temperaturas elevadas sobre o desempenho (SMITH \& TEETER, 1993). Suplementando 0,50 e $1,00 \%$ de $\mathrm{KCl}$ na ração de frangos de corte criados durante o verão, BORGES (1997) concluiu que o ganho de peso melhorou $(\mathrm{P}<0,05)$ em 3,5\%.

$\mathrm{O} \mathrm{NaHCO}_{3}$ tem sido usado pela indústria avícola na tentativa de minimizar as perdas por estresse calórico, particularmente durante o verão. Resultados de desempenho mostraram que o fornecimento de 0,5 e $1,0 \%$ de $\mathrm{NaHCO}_{3}$ em rações de frangos de corte, submetidos a temperaturas variando de 39 a $41^{\circ} \mathrm{C}$ e $34 \mathrm{a} 36^{\circ} \mathrm{C}$, proporcionou uma tendência para melhorar o consumo de ração, ganho de peso e conversão alimentar (FISCHER DA SILVA et al., 1994). BORGES (1997), suplementando 0,5; 1,0 e 1,5\% de $\mathrm{NaHCO}_{3}$ na ração de frangos durante o verão, não observou efeito sobre o desempenho.

O ganho de peso de frangos de corte é melhorado após a suplementação de $\mathrm{KCl}, \mathrm{NaCl}$ e $\mathrm{K}_{2} \mathrm{SO}_{4}$, via água ou ração, apesar do equlíbrio ácidobase das aves estar alterado. A adição de $\mathrm{NH}_{4} \mathrm{Cl}$ a para restaurar o $\mathrm{pH}$ do sangue a valores normais, não teve efeito sobre o desempenho das aves (TEETER \& BELAY, 1996).

\section{Aplicabilidade da teoria do balanço eletrolítico}

O meio ambiente e a dieta influenciam o equilíbrio ácido-base. Inúmeros relatos apontam para os efeitos do balanço eletrolítico da dieta sobre o desempenho produtivo das aves. A manutenção deste equilíbrio pode ser uma medida importante para melhorar o desempenho de frangos criados sob altas 
temperaturas e superar os efeitos danosos da alcalose respiratória decorrente do estresse calórico.

Dietas formuladas com altos teores de $\mathrm{Cl}$ $\left(\mathrm{NH}_{4} \mathrm{Cl}, \mathrm{HCl}\right.$ e $\left.\mathrm{CaCl}_{2}\right)$ diminuem o $\mathrm{pH}$ sangüíneo em frangos, prejudicando o seu crescimento em condições de termoneutralidade. Porém, o consumo de ácidos ou bases, balanço eletrolítico, ambiente, suas interações e implicações sobre o desempenho dos animais, ainda não estão bem definidas.

O estresse calórico, além de espoliar grande quantidade de ácido orgânico, pode estar associado às perdas de eletrólitos através das membranas celulares (FISCHER DA SILVA et al., 1994). A prevenção do desequilíbrio eletrolítico pode ser obtida pela incorporação de cátions e ânions na dieta, sendo usualmente expressos em mEq/kg (MONGIN, 1981). Entretanto, a disponibilidade dos eletrólitos pode ser influenciada pela regulação homeostática intestinal e renal, pela maior absorção de íons monovalentes.

Alguns autores descreveram equações para explicar a relação entre cátions e ânions e o equilíbrio ácido-base. Para MELLIERE \& FORBES (1966) esta interrelação pode ser descrita pela equação :

Nível relativo $=\frac{m E q \text { cátions }}{m E q \text { ânions }}=\frac{C a+M g+N a+K}{P O+C l+S_{4}}$

Entretanto, os íons essenciais à manutenção do equilíbrio ácido-base são: $\mathrm{Na}^{+}, \mathrm{K}^{+}$e $\mathrm{Cl}^{-}$. Além das aves os exigirem em quantidades mínimas em sua alimentação, para satisfazer suas necessidades nutricionais, é fundamental que a proporção entre eles seja ideal para manter a homeostase ácido-base e obter o máximo desempenho das aves (MONGIN, 1981). Para manter o equilíbrio ácido-base, a ave deve regular a ingestão e a excreção de ácidos. Existem diferenças na ingestão e excreção de ânions e cátions da dieta. Porém, os ácidos produzidos no metabolismo $\left(\mathrm{H}^{+}\right.$endógeno $)$também contribuem para o balanço ácidobase. Nas situações em que o animal se encontra em balanço ácido-base constante, sem excesso ou deficiência de ácido ou base, pode ser descrita a equação:

(Ânions - Cátions $)_{\text {ingeridos }}+\mathrm{H}^{+}$endógeno $=(\text {Ânions - Cátions })_{\text {excretados }}$ De acordo com MONGIN (1981) o resultado do poder ácido da ingestão de $\mathrm{Na}^{+}+\mathrm{K}^{+}-$ $\mathrm{Cl}^{-}$, é igual a diferença de cátions e ânions excretados

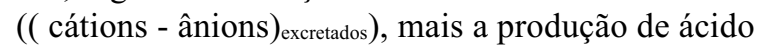
endógeno $\left(\mathrm{H}^{+}\right.$endógeno $)$, mais as bases em excesso (BEecf) ou reservas alcalinas. A ingestão ótima de eletrólitos, em termos de equilíbrio ácido-base, pode minimizar a presença de BEecf, tendendo a zero. $\mathrm{O}$ requerimento ótimo de balanço de eletrólitos foi definido em termos de $\mathrm{mEq}\left(\mathrm{Na}^{+}+\mathrm{K}^{+}-\mathrm{Cl}^{-}\right) / \mathrm{kg}$ de ração em torno de $250 \mathrm{mEq} / \mathrm{kg}$.

$(\text { Ânions }- \text { Cátions })_{\text {ingeridos }}=(\text { Ânions }- \text { Cátions })_{\text {excretados }}+$ $\mathrm{H}^{+}$endógeno + BEecf

ou,

$\left(\mathrm{Na}^{+}+\mathrm{K}^{+}-\mathrm{Cl}^{-}\right)_{\text {ingeridos }}=(\hat{\text { Ânions }}-\text { Cátions })_{\text {excretados }}+\mathrm{H}^{+}$endógeno + BEecf

$$
\mathrm{mEqNa}+\mathrm{mEqK}-\mathrm{mEqCl}=250
$$

Exemplo: $\quad 0,35 \% \mathrm{Na} \times 10000 / 23=152 \mathrm{mEq}$ $\mathrm{Na}$

$0,68 \% \mathrm{~K} \times 10000 / 39,1=174 \mathrm{mEq} \mathrm{K}$
$0,30 \% \mathrm{Cl} \times 10000 / 35,5=84 \mathrm{mEq} \mathrm{Cl}$

$152+174-84=242 \mathrm{mEq} / \mathrm{kg}$ de ração considerados:

Nestas equações, alguns fatores devem ser

a) $\mathrm{Na}$ equação, assume-se que somente os minerais $\mathrm{Na}, \mathrm{K}$ e $\mathrm{Cl}$ têm impacto no equilíbrio ácidobase sem considerar a forma que são ingeridos. A suplementação de sódio e potássio aumenta o pH e o $\mathrm{HCO}_{3}{ }^{=}$sangüíneo, enquanto que a adição de cloro deprime estes mesmos parâmetros (HURWITZ et al., 1973). Porém, há evidências de que ânions metabolizáveis exercem influência no balanço ácidobase. GORMAN \& BALNAVE (1994) concluíram que o ganho de peso associado com o carbonato de sódio e o bicarbonato de sódio foram significativamente diferentes em dietas com o mesmo balanço eletrolítico, concluindo que o estresse calórico pode induzir um requerimento metabólico para o íon bicarbonato;

b) A equação não leva em consideração os efeitos específicos de cada íon, bem como as exigências individuais destes íons o que pode limitar o uso da mesma. Lembrando que nem sempre estes íons são quantificados nos ingredientes, sendo que o $\mathrm{K}$ está presente em abundância na maioria dos ingredientes que compõem as dietas destinadas as aves, ao passo que o $\mathrm{Na}$ está presente em pequenas quantidades. As recomendações nutricionais destes eletrólitos para frangos de corte são variadas porém, o NRC (1994) recomenda 0,30,0,20 e 0,20;0,30, 0,15 e $0,15 \%$ para $\mathrm{K}, \mathrm{Na}$ e $\mathrm{Cl}$ de 0 a 3 e 3 a 6 semanas de idade, respectivamente;

c) Apesar de outros cátions e ânions também participarem no balanço ácido-base, esses não são considerados nesta equação devido a sua importância secundária. Pois, o potencial eletrolítico dos elementos pode classifica-los em termos de importância no equilíbrio ácido-base do organismo. 
Por exemplo, o K, $\mathrm{Na}$ e $\mathrm{Cl}$ têm maior potencial eletrolítico que $\mathrm{Mg}, \mathrm{S}, \mathrm{P}$ e $\mathrm{Ca}$, sendo que potencial eletrolítico destes é maior que $\mathrm{Fe}, \mathrm{Mn}, \mathrm{Zn}, \mathrm{Cu}, \mathrm{Se}$, Mo, Co e I. Os elementos traços têm capacidade de funcionar como eletrólitos mas, eles estão presentes em pequenas quantidades nas rações e em baixas concentrações nos tecidos das aves o que, naturalmente, reduz seu impacto sobre o equilíbrio ácido-básico e balanço eletrolítico nas aves. Assim, a equação eletrolítica completa seria: $\left(\mathrm{Na}^{+}+\mathrm{K}^{+}+\mathrm{Ca}^{++}\right.$ $\left.+\mathrm{Mg}^{++}\right)-\left(\mathrm{Cl}^{-}+\mathrm{SO}_{4}=+2 \mathrm{PO}_{4}=+\mathrm{HPO}_{4}^{-}\right)(\mathrm{BORGES}$, 2001).

d) Em relação aos elementos não considerados na equação resumida de MONGIN (1981) pode-se dizer que: cátions bivalentes não são rapidamente absorvidos como os cátions monovalentes; o magnésio ( $\mathrm{Mg}$ ) é comumente fornecido nas rações; o fosfato é difícil de ser quantificado, devido ser oriundo de várias fontes; a taxa de absorção do cálcio é controlada pelo sistema endócrino; o sulfato está presente em pequenas quantidades, estando relacionado com a prevenção do catabolismo da metionina.

e) Interrelação entre íons minerais e outros nutrientes, como entre $\mathrm{Na}$ e $\mathrm{Cl}$ e a relação arginina:lisina em frangos estressados pelo calor (BRAKE et al., 1998). Em frangos entre 3 e 7 semanas de idade criados em estresse calórico, a relação arginina:lisina ótima foi 1,34, quando $\mathrm{Na}$ e $\mathrm{Cl}$ foram mantidos nas recomendações do NRC (1994). Entretanto, quando os teores de $\mathrm{Na}$ e $\mathrm{Cl}$ na dieta foram aumentados a relação arginina:lisina ficou abaixo de 1,05 , mostrando que em situações de estresse calórico o ajuste do balanço eletrolítico para o máximo desempenho das aves pode estar relacionado à composição de aminoácidos da dieta.

HURWITZ (1981), ao contrário de MONGIN (1981), propôs que o balanço entre $\mathrm{Na}^{+} \mathrm{e}$ $\mathrm{Cl}^{-}$é que determina primariamente a concentração de $\mathrm{HCO}_{3}^{-}$e o $\mathrm{pH}$ do plasma. $\mathrm{O} \mathrm{K}^{+}, \mathrm{Ca}^{++}$e $\mathrm{Mg}^{++}$ compõem os outros cátions e o $\mathrm{HCO}_{3}^{-}$, as proteínas e outros ânions em baixais concentrações (fosfato, sulfato, lactato e piruvato) são os ânions do plasma. $\mathrm{O} \mathrm{HCO}_{3}^{-}$e as proteínas (incluindo a hemoglobina) constituem os tampões básicos, e assim como as BEecf são considerados componentes metabólicos do equilíbrio ácido-base. As BEecf expressam a quantidade de ácidos ou bases que, quando adicionadas em um litro de sangue, retornam o $\mathrm{pH}$ ao normal. O tampão básico é considerado por ROOTH (1969) como uma junção entre equilíbrio ácido-base e balanço de eletrólitos. $\mathrm{O}$ íon $\mathrm{H}^{+}$não está presente no diagrama, uma vez que exerçe influência em menores concentrações do que o $\mathrm{Cl}^{-}, \mathrm{K}^{+}$e outros íons.

Com a variação dos níveis de $\mathrm{Na}^{+}$e $\mathrm{Cl}^{-}$na ração, observou-se melhor crescimento das aves quando a relação $\mathrm{Na}: \mathrm{Cl}$ foi de aproximadamente 1:1, com a utilização de rações contendo $\left(\mathrm{Na}^{+}+\mathrm{K}^{+}-\mathrm{Cl}^{-}\right)$a $200 \mathrm{mEq} / \mathrm{kg}$ (HURWITZ et al.,1973). As alterações no equilíbrio ácido-base e desequilíbrios na suplementação de $\left(\mathrm{Na}^{+}+\mathrm{K}^{+}-\mathrm{Cl}^{-}\right)$, causam inapetência, com redução no ganho de peso, prejudicando a conversão alimentar, queda da produção de ovos e quando os desequilíbrios não são compensados determinam aumento na mortalidade (MONGIN, 1981). Nas aves em alcalose, a concentração sangüínea dos eletrólitos $\left(\mathrm{Na}^{+}, \mathrm{K}^{+}\right)$está diminuída. Uma redução no estado de alcalose ocorre, quando a relação $\mathrm{Na}: \mathrm{Cl}$ diminui, adicionando-se 0,5 e 1,0\% de $\mathrm{CaCl}_{2}$, melhorando $8,0 \%$ o desempenho das aves (TEETER et al., 1985).

JOHNSON \& KARUNAJEEWA (1985) concluíram que um balanço de eletrólitos na dieta menor que $180 \mathrm{mEq} / \mathrm{kg}$ e maior que $300 \mathrm{mEq} / \mathrm{kg}$ deprimiu o peso das aves, quando avaliado aos 42 dias de idade. Um ótimo balanço eletrolítico foi encontrado para rações contendo de 250 a $300 \mathrm{mEq} / \mathrm{kg}$. Do mesmo modo, HULAN et al. (1987), pesquisando o efeito de rações contendo $\mathrm{Na}^{+}+\mathrm{K}^{+}-\mathrm{Cl}^{-}$em diferentes proporções e ainda variando o nível de cálcio, constataram que o pior e o melhor ganho de peso foram obtidos quando o "número de MONGIN" foi de 174 e $215 \mathrm{mEq} / \mathrm{kg}$, com 1,38 e $0,95 \%$ de cálcio, respectivamente.

BORGES et al., (1999) observaram que o melhor balanço eletrolítco variou com o eletrólito manipulado, o melhor ganho de peso foi observado quando as dietas apresentavam $199 \mathrm{mEq} / \mathrm{kg}$ e os íons $\mathrm{Na}^{+} \mathrm{e} \mathrm{Cl}-$ foram manipulados. Os autores recomendam que níveis extremos de $\mathrm{Cl}^{-}(0,15$ e $0,71 \%), \mathrm{K}^{+}(0,98$ e $1,21 \%)$ e $\mathrm{Na}^{+}(0,15$ e $0,60 \%)$ devem ser evitados em dietas pré-iniciais. RONDÓN et al. (2000) também observaram uma variação no melhor balanço eletrolítico de acordo com o íon manipulado. O melhor balanço eletrolítico foi de $250 \mathrm{mEq} / \mathrm{kg}$ quando os níveis de $\mathrm{Na}^{+}$variaram e $319 \mathrm{mEq} / \mathrm{kg}$ quando o íon manipulado foi o $\mathrm{K}^{+}$.

A fonte protéica utilizada na ração pode afetar o equilíbrio eletrolítico e ácido-base, pois certas fontes, principalmente as de origem animal, aumentam a produção de ácidos orgânicos e reduzem a contribuição de $\mathrm{Na}$ e K, aumentando a quantidade relativa de $\mathrm{Cl}$ (PORTSMOUTH, 1984). O fornecimento de rações basicamente constituídas por farelo de soja, que contêm baixos teores de $\mathrm{Na}$ e altos 
em $\mathrm{K}$, demonstrou uma resposta significativa no desenvolvimento de frangos de corte, suplementados com 0,5 e 1,0\% de $\mathrm{NaCl}(\mathrm{MARCH}, 1984)$.

$\mathrm{O}$ impacto da relação cátion/ânion sobre o equilíbrio ácido-base de frangos, $\mathrm{pH}$ sangüíneo e taxa de crescimento foi avaliada por HURWITZ et al. (1973). A taxa de crescimento de frangos foi máxima quando o pH sangüíneo era de 7,28, sendo observado um declínio no crescimento quando os valores $\mathrm{pH}$ foram acima de 7,30 ou abaixo de 7,20. A relação eletrolítica da dieta para o máximo crescimento variou de 226 a 260 $\mathrm{mEq} / \mathrm{kg}$. Entretanto, se a resposta é devido inteiramente às alterações no $\mathrm{pH}$ ou a outros efeitos eletrolíticos ou metabólicos, não está bem claro. TEETER et al. (1985) indicaram que durante o período de ofegação, os valores de $\mathrm{pH}$ acima de 7,25 deprimem a taxa de crescimento e a eficiência alimentar. Aumentos no $\mathrm{pH}$ sangüíneo podem ser reduzidos por uma diminuição da taxa respiratória das aves. Estes aumentos podem ocorrer com temperaturas de aclimatação mas, durante o estresse agudo, a taxa respiratória pode ser reduzida e a eficiência da perda de calor pode ser melhorada pela manutenção da alta ingestão de água (BELAY \& TEETER, 1993). A mortalidade durante o estresse calórico pode ser inversamente relacionada com o consumo de água (BRANTON et al., 1986).

BORGES (2001) concluiu que a resposta ao balanço eletrolítico da dieta depende da temperatura ambiente. A ingestão de água está na dependência direta da idade da ave e da relação $\mathrm{Na}+\mathrm{K}-\mathrm{Cl}$ na ração, sendo que o aumento na ingestão de água provocado pela maior relação $\mathrm{Na}+\mathrm{K}-\mathrm{Cl}$ afeta diretamente a umidade da cama e reduz a temperatura retal nas aves. A relação eletrolítica da dieta interfere no desempenho das aves, sendo que a relação ideal variou de 186 a $250 \mathrm{mEq} / \mathrm{kg}$. Balanço eletrolítico elevado (340 e $360 \mathrm{mEq})$ pode resultar em alcalose metabólica. Em estresse calórico, a ave retém mais eletrólitos $(\mathrm{Na}, \mathrm{K} \mathrm{e} \mathrm{Cl})$ na tentativa de manter o equilíbrio ácido base. A quantidade de eletrólitos excretados via urinária depende da concentração destes na ração e da temperatura ambiente.

\section{CONCLUSÕES}

A exposição de frangos de corte a temperatura ambiente elevada resulta em alcalose respiratória, provocando queda de desempenho zootécnico. A formulação de rações com base no conceito de balanço eletrolítico, bem como a adição de sais na água e/ou na ração são práticas que podem ser implementadas para corrigir distorções no equilíbrio ácido-base decorrentes do estresse calórico.

\section{REFERÊNCIAS BIBLIOGRÁFICAS}

AIT-BOUlahsen, A.; GARLiCH, J. D.; EDENS, F.W. Potassium chloride improves the thermotolerance of chickens exposede to acute heat stress. Poultry Science, v.74, p.75-87, 1995.

BACILA, M. Bioquímica veterinária. São Paulo : Varela, 1980. 534 p.

BELAY, T.; TEETER, R.G. Broiler water balance and thermobalance during thermoneutral and high ambient temperature exposure. Poultry Science, v.72, p.116-124, 1993.

BORGES, S.A. Suplementação de cloreto de potássio e bicarbonato de sódio para frangos de corte durante o verão. 1997. 84f. Dissertação (Mestrado em Zootecnia)-Curso de Pósgraduação em Zootecnia, Universidade Estadual Paulista.

BORGES, S.A. Balanço eletrolítico e sua interrelação com o equilíbrio ácido-base em frangos de corte submetidos a estresse calórico. 2001. 97f. Tese (Doutorado em Zootecnia) - Curso de Pós-graduação em Zootecnia, Universidade Estadual Paulista.

BORGES, S.A. et al. Balanço eletrolítico em dieta pré-inicial de frangos de corte durante o verão. Revista Brasileira de Ciência Avícola, v.1, p.175-179, 1999.

BRAKE, J.; BALNAVE, D.; DIBNER, J.J. Optimum dietary arginine:lysine ratio for broiler chickens is altered during heat stress in association with changes in intestinal uptake and dietary sodium chloride. British Poultry Science, v.39, p.639-647, 1998.

BRANTON, S.L.; REECE, F.N.; DEATON, J.W. Use of ammonium chloride and sodium bicarbonate in acute heat exposure of broilers. Poultry Science, v.65, p.1659-1663, 1986.

CUNNINGHAM J.G. Tratado de fisiologia veterinária. Rio de Janeiro : Guanabara, 1999. 454p.

FISCHER DA SILVA, A.V.; FLEMMING, J.S.; FRANCO, S.G. Utilização de diferentes sais na prevenção do estresse calórico de frangos de corte criados em clima quente. Revista Setor de Ciências Agrárias, v.13, p.287-292, 1994.

GORMAN, I.; BALNAVE, D. Effects of dietary mineral supplementation on the performance and mineral retentions of broilers at high ambient temperatures. British Poultry Science, v.35, p.563-572, 1994.

HULAN, H.W.; SIMONS, P.C.M.; VAN SCHAGEN, P.J.W. Effect of altering the cation-anion $(\mathrm{Na}+\mathrm{K}-\mathrm{Cl})$ and calcium content of the diet on general performance and incidence of tibial dischondroplasia of broiler chickens housede in batteries. Nutrition Reports International, v.33, p.397-408, 1987.

HURWITZ, S.; COHEN, I.; BAR, A. Sodium and chloride requeriments of chick: relationship to acid-base balance. Poultry 
Science, v.52, p.903-909, 1973.

HURWITZ, S. Requirements and interaction of monovalant ions in nutrition. In: ANNUAL MINERALS CONFERENCE, 4., 1981, Mundelein/IL. Proceedings... Mundelein : n.i., 1981. p.27-35.

JOHNSON, R. J.; KARUNAJEEWA, H. The effects of dietary minerals and electrolytes on the growt and physiology of the young chick. Journal of Nutrition, v.115, p.1680-1690, 1985.

MACARI, M.; FURLAN, R.L.; GONZALES, E. Fisiologia aviária aplicada a frangos de corte. Jaboticabal : FUNEP/ UNESP, 1994. 246p.

MARCH, B.E. Sodium chloride supplementation of all plant protein broiler diets. Poultry Science, v.63, p.703-705, 1984.

MELLIERE, A. L.; FORBES, R.M. Effect of altering the dietary cation-anion ratio on food consumption and growth of young chicks. Journal of Nutrition, v.90, p.310-314, 1966.

MONGIN, P. Recent advances in dietary cation-anion balance: applications. In: POULTRY PROCEEDINGS NUTRITION SOCIETY, 1981, Cambridge. Proceedings... Cambridge : n.i., 1981. V.40, p.285-294.

NOSE, H. et al. Role of osmolality and plasma volume during dehydration in humans. Journal Applied Physiology, v.65, p.325331, 1988.
NRC (NATIONAL RESEARCH COUNCIL). Nutrient requirements of poultry. Washington : National Academy, 1994. 155p.

PORTSMOUTH, J. Changes needed in nutrient input data relating to leg problems in poultry. Feedstuffs, v.56, p.43-52, 1984.

RONDÓN, E.O.O. et al. Exigências nutricionais de sódio e cloro e estimativa do melhor balanço eletrolítico da ração para frangos de corte na fase pré-inicial (1-7 dias de idade). Revista Brasileira de Zootecnia, v.29, p.1162-1166, 2000.

ROOTH, G. Introduction to acid-base and electrolyte balance. New York : Barnes and Noble, 1969.

SALVADOR, D. et al. Suplementação de bicarbonato de sódio na ração e na água de bebida de frangos de corte submetidos ao estresse calórico. ARS Veterinária, v.15, p.144-148, 1999.

SMITH, M.O.; TEETER, R.G. Carbon dioxide, ammonium chloride, potassium chloride, and performance of heat distressed broilers. Journal of Applied Poultry Research, v.2, p.61-66, 1993.

TEETER, R.G.; BELAY, T. Broiler management during acute heat stress. Animal Feed Science and Techonology, v.58, p.127-142, 1996.

TEETER, R. G. et al. Chronic heat stress and respiratory alkalosis: ocurrence and treatement in broiler chicks. Poultry Science, v.64, p.1060-1064, 1985. 